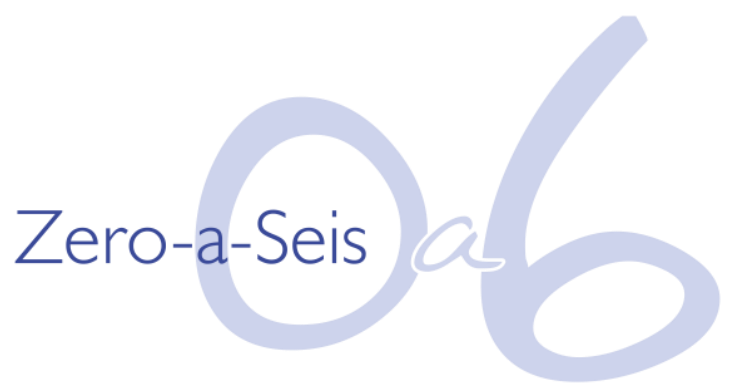

\title{
DE REGRESO A CASA: HOMESCHOOLING VERSUS EDUCACIÓN ESCOLAR
}

Back home: Home education versus school education

De volta para casa: ensino doméstico versus educação escolar

\author{
Dora Lilia MARÍN-DÍAZ \\ Facultad de Artes \\ Universidad Distrital Francisco José de Caldas \\ Bogotá, Colombia \\ dlmarind@udistrital.edu.co \\ https://orcid.org/0000-0002-0466-3368
}

\author{
Ana Cristina LEÓN-PALENCIA \\ Facultad de educación \\ Universidad Pedagógica Nacional \\ Bogotá, Colombia \\ acleon@pedagogica.edu.co \\ https://orcid.org/0000-0001-7489-0681
}

\section{RESUMEN}

A lista completa com informações dos autores está no final do artigo

El año 2020 nos recordó la fragilidad humana debido a los efectos de la pandemia generada por el virus Sars-Cov-2 y que se conoce por el nombre de la enfermedad que genera: Covid-19. La cuarentena declarada en muchos países para mitigar el contagio, generó transformaciones en la manera de llevar a cabo la educación formal, la principal de ellas es su desarrollo en aulas remotas, lo que reforzó la idea de la enseñanza doméstica o el Homeschooling como una alternativa a la educación escolar. En esta dirección, en este articulo se argumenta que si bien algunas de las transformaciones sufridas en las prácticas escolares durante este tiempo quizá modifiquen los modos de hacer escuela, también es cierto que la forma escuela es un cronotopo, un espacio-tiempo que permite a los niños y jóvenes la construcción de herramientas para acceder al mundo y construir mundos posibles.

PALABRAS CLAVES: Homeschooling. Escolarización. Educación en casa. Escuela.

\begin{abstract}
The year 2020 reminded us of human fragility due to the effect of the pandemic generated by the SarsCov- 2 virus, which is known by the name of the disease it generates: Covid-19. The quarantine declared in many countries to mitigate contagion brought transformations in the way of carrying out formal education, the main one being the development of remote classes that reinforced the idea of home education or Homeschooling as an alternative to school education. Thus, the article argues that even if it is still certain that some of the transformations undergone in school practices at this time will change the ways of doing school, it is also certain that the school form is a chronotope, a space-time that allows children and young people the setting up of tools to access the world and build possible worlds.
\end{abstract}

KEYWORDS: Homeschooling. Schooling. Home education. School.

\section{RESUMO}

O ano de 2020 lembrou-nos da fragilidade humana pelo efeito da pandemia gerada pelo vírus Sars-Cov2 , e que se conhece pelo nome da doença que ele gera: o Covid-19. A quarentena declarada em muitos países para mitigar o contágio trouxe transformações na forma de realizar a educação formal, a principal delas o desenvolvimento de aulas remotas que reforçaram a ideia do ensino doméstico ou Homeschooling como uma alternativa para o ensino escolar. Nessa direção, no artigo argumenta-se que ainda seja certo que algumas das transformações sofridas nas práticas escolares neste tempo vão mudar os modos de fazer escola, também é certo que a forma escola é um cronotopo, um espaço-tempo que permite às crianças e jovens a construção de ferramentas para acessar o mundo e construir mundos possíveis.

PALAVRAS-CHAVE: Homeschooling. Educação escolar. Educação domiciliar. Escola. 


\section{INTRODUCCIÓN}

La educación universal por medio de la escolarización no es factible. No sería más factible si se intentara mediante instituciones alternativas construidas según el estilo de las escuelas actuales. Ni nuevas actitudes de los maestros hacia sus alumnos, ni la proliferación de nuevas herramientas y métodos físicos o mentales (en el aula o en el dormitorio), ni, finalmente, el intento de ampliar la responsabilidad del pedagogo hasta que englobe las vidas completas de sus alumnos, dará por resultado la educación universal. La búsqueda actual de nuevos embudos educacionales debe revertirse hacia la búsqueda de su antípoda institucional: tramas educacionales que aumenten la oportunidad para que cada cual transforme cada momento de su vida en un momento de aprendizaje, de compartir, de interesarse (ILLICH, 2011, p.189).

La presencia del virus Sars-Cov2 y sus efectos sobre la salud, identificados a finales de 2019 en la ciudad de Wuhan (China), la posterior declaración de pandemia emitida por la Organización Mundial de la Salud (OMS) el 11 de marzo de 2020, y el aumento en el número de contagios y muertes que se registraba diariamente en un creciente número de países alrededor del mundo, llevaron a los distintos gobiernos al establecimiento de medidas excepcionales. En los primeros meses, la sorpresa, el desconcierto y el temor orientaron la toma de decisiones centradas en el confinamiento y la restricción de la movilidad de amplios sectores de la población por parte de los gobernantes y marcaron la reacción de los sujetos ante la interrupción de la denominada "normalidad" en la vida social, familiar y personal.

Este evento de alcance mundial se puede leer como el gesto de un acontecimiento en el que se interrogan las formas de vida actuales en sus múltiples dimensiones, un acontecimiento en la medida en que se inquiere el presente, lo que hacemos, las formas como pensamos y actuamos al dejar en suspenso los modos de vivir considerados normales. En esos primeros meses, en distintos países, aunque no en todos, el Estado aparecía como la institución que podía y debía atender, cuidar y resolver las necesidades de sus habitantes; entonces, pareció renovarse la confianza en la institucionalidad estatal y en la capacidad de los gobernantes. Las medidas restrictivas de la movilidad limitaron la asistencia a las oficinas, al trabajo, a las escuelas, a las universidades, a los espacios sociales y a las zonas comerciales, entre otros, aceptándose como necesarias para preservar la vida; sin embargo, no pasaron muchas semanas para que una reacción contraria apareciera.

Al término de los primeras semanas de confinamiento y ante el anuncio de nuevas restricciones y de un aislamiento social que se mantendría durante los siguientes meses, hasta tener acceso a alguna de las vacunas que se estaban ensayando para alcanzar la "inmunidad de rebaño" y detener la proliferación del virus, 
se ampliaron las manifestaciones de inconformidad, no solo porque se perdieron empleos, porque el comercio formal e informal se interrumpió o funcionó de manera intermitente, sino porque con ello se hizo evidente la fragilidad de los sistemas económicos y la pobreza en la que vive buena parte de la población mundial. Además, porque las personas no estaban preparadas para permanecer 24 horas, siete días, a la semana en un espacio solitario o compartido con todos los integrantes de su grupo familiar; porque los espacios de habitación no estaban adaptados para que allí se desarrollaran simultáneamente actividades de estudio, trabajo, recreación, socialización, etc.; y porque la transformación de los hábitos cotidianos ocurrió de manera acelerada y modificó los modos como cada uno asumió las formas que tomaron las relaciones familiares, laborales y estudiantiles. Finalmente, porque se difundieron críticas a las decisiones de confinamiento, a la restricción de movilidad y al control de las tareas y actividades diarias decretados por los gobiernos locales y nacionales; estas empezaron a leerse como limitadoras de las libertades individuales y se generaron sospechas sobre la concentración de poderes en los gobernantes, durante el "Estado de excepción" que rompía el equilibrio de poderes que caracteriza las democracias. En estas condiciones ganaron terreno teorías conspirativas y "negacionistas" que, hasta hoy, ven complots e intereses oscuros en la forma como apareció el virus, en el manejo de epidemia, en la creación y aplicación de las vacunas, etc.

En otras palabras, el debate sobre la salud económica de los Estados, de las familias y de los individuos se cruza hoy con el debate sobre la salud física y psicológica de los individuos y los núcleos familiares sometidos a aislamientos prolongados. Estudios y estadísticas muestran problemáticas de diverso tipo: el incremento en la violencia intrafamiliar, tanto entre adultos como hacia los niños y adolescentes; la disminución en la productividad laboral -debido, entre otras cosas, al aumento de actividades, la extensión del horario de trabajo y la atención simultánea de tareas-; el crecimiento de la deserción escolar, aunado a la sospecha sobre la baja calidad en la formación recibida por los estudiantes y ofrecida por los profesores como efecto de los problemas en la conectividad, la falta de herramientas y equipamientos tecnológicos, las dificultades para adaptarse y adoptar metodologías de enseñanza para el aprendizaje efectivo de los estudiantes. Entonces, si en un primer momento prevaleció el miedo, pero también la confianza en un retorno rápido a la "normalidad" que acompañó la aceptación de las medidas gubernamentales para el control de la pandemia, en el segundo momento, la extensión de la situación, sumada 
a la insatisfacción, al temor, a la sospecha, al cansancio, al dolor, al hambre, entre otras sensaciones y afectaciones, actuaron como detonantes para una serie de quejas y reclamos que ponen en cuestión la forma como nos estamos conduciendo y como estamos aceptando la conducción de los gobernantes de nuestras ciudades y países en la condición actual.

Uno de los asuntos que se cuestiona con frecuencia y que cada vez recibe más atención es la suspensión de las clases presenciales en escuelas y universidades. En el comienzo de la pandemia, el uso de plataformas como Meet y Zoom apareció como una interesante solución para los encuentros grupales, mientras que la aplicación Whatsapp y las herramientas de Google, entre otras, funcionaron para realizar seguimientos individuales y acompañamiento a las tareas y actividades. Aunque profesores y estudiantes no estaban habituados a la "educación remota" -que en ningún caso debía entenderse como educación a distancia (EAD), un modelo que venía funcionando desde hace varias décadas, con metodologías y herramientas diseñadas para tal fin-, esta se propuso como una alternativa pasajera que suplía parcialmente el proceso que ocurría en la escuela de forma presencial. Al respecto, la percepción de este cambió osciló entre dos perspectivas: por un lado, hubo varias críticas frente a la transformación y novedad que supusieron la educación remota y el uso de herramientas tecnológicas para su desarrollo, debido a problemas en la cobertura de Internet y el acceso a dispositivos electrónicos - problema especialmente en las zonas rurales y los sectores socio-económicos marginales-, pero también por la falta de preparación de estudiantes, profesores y cuidadores (padres, hermanos, abuelos, etc.) para realizar actividades utilizando tales medios, lo que en algunos casos generó importantes cargas de estrés y preocupaciones por la salud mental. Por el otro lado, hubo quienes celebraron la aparición de esta alternativa, pues se le vio como una oportunidad para la actualización de la enseñanza escolar e inclusive como una evidencia sobre la pertinencia del homeschooling como alternativa a la educación escolar (criticada por tradicional, obsoleta, poco efectiva, etc.).

Estas últimas manifestaciones nos llevaron a proponer y argumentar a favor de una hipótesis: si bien es cierto que, con la "nueva normalidad" la escuela está compelida a sufrir transformaciones o actualizaciones, con ella también se hizo evidente que la forma escuela (en sus diferentes niveles o grados de educación formal) ofrece un espacio y un tiempo irremplazables para el estudio, el ejercicio, el ensayo y la construcción de herramientas que aproximan a los niños y a los jóvenes a un mundo que de otra forma no sería accesible para ellos. El espacio-tiempo escolar 
es un cronotopo en el que se adquieren unas experiencias de formación que no se consiguen de otra forma, aunque por fuera de la escuela ocurran los mas variados aprendizajes y experiencias. La enseñanza, el saber, la experiencia que ofrece ese cronotopo es una parte fundamental de la formación; tal vez esa sea una experiencia sin la cual se puede vivir, sin embargo, es necesario que nos preguntemos ¿qué es lo que se pierde cuando no se tiene la oportunidad de habitar ese espacio y vivir las experiencias que allí se producen? ¿por qué los infantes y los jóvenes exigen el retorno a las clases presenciales? ¿la educación a distancia, la virtualidad, la educación en casa son alternativas a la educación escolar?

\title{
A PROPÓSITO DEL HOMESCHOOLING
}

\begin{abstract}
Las escuelas son todavía menos eficientes en la creación de circunstancias que alienten el uso irrestricto, exploratorio, de habilidades adquiridas, para lo cual reservé el término de "educación liberal". El principal motivo es que la escuela es obligatoria y llega a convertirse en la escolaridad por la escolaridad: una estadía forzosa en compañía de profesores, que paga con el dudoso privilegio de continuar en dicha compañía. Así como la instrucción de destrezas debe ser liberada de restricciones curriculares, a la educación liberal debe desligársela de la asistencia obligatoria. Mediante dispositivos institucionales puede ayudarse tanto al aprendizaje de habilidades como a la educación encaminada a un comportamiento creativo e inventivo, pero ambas cosas son de naturaleza diferente y frecuentemente contraria. (ILLICH, 2011, p.206).
\end{abstract}

En 1976 John Holt publicó Instead of Education: Ways to Help People do Things Better, un texto que sintetizó algunos aspectos del espíritu del homeschooling, entre los que señaló que siendo el principal derecho humano el de controlar la propia mente y sus pensamientos, era necesario defender el derecho a explorar el mundo, los pensamientos propios y los de otras personas, así como adquirir experiencias que permitn encontrarle sentido a la propia vida. la contracara de este propósito sería el proceso de escolarización, pues correspondería a un sistema compulsivo y competitivo se privilegian los estímulos, la posibilidad de destacar, los grados, los diplomas y las credenciales. De esta forma, la educación escolar se caracterizaría como una práctica que niega el derecho humano de hacer un modelaje autónomo de sí mismo.

En una perspectiva similar, los trabajos de Illich apuntan a una educación liberal, en la que se renuncia a las características propias de la educación escolar, en especial a su obligatoriedad. Illich propone, en contra de los establecimientos educacionales especializados, la organización de redes conformadas por diversos actores sociales, agrupados de manera voluntaria, focalizada y flexible que ayuden en la generación de diversos procesos de aprendizaje. Con esta propuesta no se busca la transformación, ajuste o reorganización de la escuela sino su eliminación y la 
producción de otras formas para llevar a cabo la educación (NARODOWSKI, BOTTA, 2017). La organización de redes y el homeschooling encuentran su común denominador en la idea de promover y garantizar aprendizajes fuera de la escuela y en generar una propuesta "educativa" en la cual los cuidadores primarios asuman (con ayuda de otras personas o instituciones sociales) la responsabilidad formativa de los infantes y jóvenes, en lo que se refiere a la adquisición de conocimientos y habilidades y a la transmisión de valores y principios culturales y sociales.

Al respecto es importante pensar que la familia y la escuela son instituciones modernas que tuvieron tareas diferenciadas respecto a la educación. Esa diferenciación se borra en estas propuestas de educación em casa, pues de cierta manera, se delega a la familia las tareas educativas que tenía la escuela, haciendo a esta última irrelevante o innecesaria. Sin embargo, también se experimenta en las sociedades actuales un movimiento en sentido contrario, pues en muchos casos y en los distintos sectores de la población, la escuela ha asumido em las últimas décadas varias funciones que le corresponderían a las familias em términos del cuidado y la protección de los sujetos infantiles.

El homeschooling o educación en casa supone el traslado de ciertas prácticas de la escolarización al escenario familiar y la promoción de actividades complementarias que amplíen las experiencias formativas de cada individuo. Junto a este planteamiento, aparecen otras dos posiciones que se pueden identificar así: la primera, más radical y crítica se conoce como unschooling, allí se sugiere una ruptura total de la forma escolar y el rechazo de cualquier perspectiva educativa que siga sus cánones; en esta propuesta la actividad de formación está determinada por los jóvenes y lo que ellos quieran aprender, en los tiempos en que deseen hacerlo. La segunda, una alternativa intermedia sugiere la flexibilización en ciertos procesos y se denomina flexischooling, en ella se apunta a la necesidad de complementar la educación en casa con la asistencia en tiempo parcial a la institución escolar (CABO, 2012).

Además de estas posiciones es importante destacar que no todos los que se refieren al homeschooling la entienden o la practican de la misma manera. Por ejemplo, las posiciones ortodoxas consideran que la casa es una escuela en miniatura y por lo tanto debe funcionar con currículos formales y convencionales; entre tanto, las posturas liberales proponen una suerte de aprendizaje "desestructurado" que se guie por las habilidades innatas de los aprendices, sin una enseñanza formal (GUTERSON, 1992). La distinción entre estas propuestas no solo es nominal, ellas 
remiten a posiciones, comprensiones y prácticas relacionadas con la educación que orientan la actividad formativa hacia caminos diferentes y en la cuales podemos percibir lecturas distintas de lo que es y hace la escuela, pero también el desconocimiento de lo que ella es y puede hacer, pues, si bien es cierto que no responde a lo que en ocasiones se le exige, también es cierto que ella ofrece algo que en ningún otro espacio se puede lograr.

Ahora bien, aunque el homeschooling es un fenómeno cada vez más extendido, su aceptación y reconocimiento normativo en los diversos países es disímil. Al respecto, la tesis titulada El homeschooling en España: descripción y análisis del fenómeno (CABO, 2012), realizada hace casi una década, cita un estudio de OIDEL, una ONG que se especializa en acciones orientadas al derecho a la educación y la libertad de enseñanza ${ }^{1}$, en el cual los países son clasificados según el grado de libertad que conceden a sus ciudadanos para definir el modelo educativo que desean seguir; lo que resulta significativo es que para hacerlo usan como referente las condiciones bajo las cuales se autoriza la educación en casa (homeschooling). Allí se describen cuatro grupos: a) Estados en los que el homeschooling es autorizado bajo mínimas condiciones de supervisión; b) Estados en los que el homeschooling es autorizado bajo estrictas condiciones de supervisión; c) Estados en los que es autorizado en casos particulares; y c) Estados en los que el homeschooling no forma parte de la política educativa, ni se reconoce. Seguramente, esta clasificación continúa vigente hoy en países de Europa y América, pero lo que llama la atención del estudio es la relevancia que el homeschooling ha ganado como alternativa para la educación escolar y, sobre todo, su vinculación como patrón para medir el "grado de libertad ciudadana" y el cumplimiento del "derecho a la educación", dos temas que paradójicamente están en juego en este momento que se exige el retorno a clases presenciales en las escuelas: libertad de movimiento, de encuentro con otros; espacios de socialización y convivencia con pares, el derecho a una educación de calidad que ofrezca más y mejores experiencias educativas, etc.

En América Latina los debates recientes sobre la reglamentación del homeschooling muestran el espacio que esta práctica ganó durante las últimas décadas. En Colombia, por ejemplo, en el año 2020 se presentó al Senado el proyecto "Ley homeschooling: Educación en Casa" (CASTELLANOS y SANCHEZ, 2000), con el cual se busca modificar la Ley General de Educación (1994) y autorizar la realización

\footnotetext{
${ }^{1}$ ONG que actúa como instancia consultiva de la UNESCO, el Consejo de Europa y las Naciones Unidas.
} 
de la educación preescolar, básica y media en casa. En estos casos los educadores pueden ejercer sus labores sin formación profesional, aunque deban capacitarse 0 profesionalizarse - no se sabe en qué o sobre qué - y buscar el apoyo de instituciones privadas para que les realicen el acompañamiento, verificación, evaluación y seguimiento del proceso educativo. Al respecto son varias las preguntas que surgen sobre las exigencias en la formación de los profesores y la supervisión que el Estado debe hacer de estos procesos como regulador del Sistema de educación formal escolar; además, después de más de un año de aulas remotas en la mayor parte del territorio nacional, se hace evidente que la enseñanza y la formación que realizan los profesores no se sustituye con el acompañamiento que los adultos responsables de los infantes y jóvenes realizan en casa.

Por su parte, para el caso brasileño, según lo descrito en la exposición de motivos del proyecto de Ley Homeschooling: Educación en Casa, el asunto se encuentra en conflicto puesto que las decisiones judiciales han ratificado que la obligatoriedad escolar sea presencial, en instituciones educativas acreditadas y se ha negado cualquier posibilidad para que la misma sea de modo virtual o en casa. Según la decisión judicial del Tribunal Supremo Federal de Brasil, la educación en casa no podía ser aceptada al no existir legislación que la regule y que establezca las reglas de este tipo de educación. ¿Qué diferencia entonces al fenómeno del homeschooling y la experiencia de la nueva normalidad, centrada en el acompañamiento que han hecho los padres en casa a la escolarización de sus hijos?

Una revisión detallada de las razones que, antes de la pandemia, condujeron a los adultos responsables de infantes y jóvenes para tomar la decisión de practicar la educación en casa, independientemente de la forma como ella sea entendida, puede informarnos sobre las apuestas que están en juego cuando se toma esta opción. Uno de los primeros estudios, realizado en la década de ochenta, del siglo pasado, señala tres motivos: reforzar los valores familiares, transmitir valores y creencias y satisfacer necesidades educativas específicas en los hijos (VAN GALEN, 1987). Una investigación posterior, en la década del noventa, identifica cinco razones: elevados costos de la enseñanza privada, buscar la cohesión de la familia, evitar las influencias negativas de los escolares, afianzar las creencias religiosas y el aprendizaje (MARSHALL Y VALLE, 1996). Por último, un estudio publicado en la primera década de este siglo, señala motivaciones asociadas con los idearios que se movilizan a través del homeschooling - libertad y flexibilidad - y otras con las experiencias y situaciones que se generan 
en los espacios escolares y que se quieren evitar - bullying, mala gestión escolar, influencias negativas, entre otros - (ROTHERMED, 2003).

En general, las motivaciones que llevan a la práctica del homeschooling se relacionan con motivos de credo o religión, con preocupaciones por el éxito académico, y con las dudas sobre los entornos relacionales que ofrece el espacio escolar (MAYBERRY, 1989). Alrededor de las motivaciones religiosas se mueven adultos con creencias que van desde el cristianismo evangélico, pasan por prácticas orientales o ancestrales, hasta los colectivos que se reconocen como de la New Age; para ellos la educación debe reproducir su modo de vida y por eso es necesario controlar los contenidos transmitidos, las experiencias que tienen los jóvenes y las relaciones que establecen con otros. Sobre los logros académicos y socio-relacionales se organizan apuestas por el éxito laboral, el desarrollo y el mejoramiento social y económico. Por último, evitar o proteger de ambientes adversos por violencia, drogas, o maltrato psicológico en el tiempo de su formación de los jóvenes, constituye uno de los argumentos más fuertes para evitar los espacios que ofrece el sistema educativo formal.

Finalmente, es necesario señalar que, si bien la educación en casa es un fenómeno en expansión, también es cierto que ella no sólo no es una opción a la cual todas las personas pueden acceder, además es una propuesta que no puede ofrecer la experiencia social y los aprendizajes que ocurren en la escuela. Al parecer la escuela es una institución, entre otras, de la sociedad con una función particular que no responde a propósitos de credo, economía o aislamiento que si ofrecería el homeschooling; la escuela es un espacio y un tiempo libre de la cotidianidad y de aspiraciones adultas y familiares particulares, es un cronotopo para un tipo específico de aprendizaje, ese que ofrece "por una parte, una iniciación al conocimiento y las habilidades y, por otra, una socialización de los jóvenes en la cultura de una sociedad" (SIMONS y MASSCHELEIN, 2014, p. 27). Con la enseñanza escolar se producen aprendizajes, a través de unas formas específicas de saber, en compañía de pares generacionales y con la guía de un adulto con quien establecen una relación diferente a la que sostiene con los otros adultos del entorno familiar; saberes, relaciones $y$ experiencias con los que ensayan, se ejercitan, conocen y construyen maneras de relación con los otros, consigo mismos y con el mundo que no los anteceden, pero que se actualizan con su presencia. 


\section{SOBRE EL CRONOTOPO ESCOLAR}

La práctica del homeschooling y la experiencia de clases remotas son dos cosas distintas. Implementar la educación en casa como proyecto de educación para los infantes y jóvenes de la familia supone la disposición de espacios, tiempos, materiales y actividades acompañadas por adultos responsables y bajo directrices que proveen los programas, las comunidades o las instituciones que acompañan una apuesta centrada en garantizar el aprendizaje. La experiencia de clases remotas como alternativa temporal a los encuentros presenciales, mientras se supera la condición de pandemia actual, significó la aproximación de adultos (padres, cuidadores, profesores) y estudiantes a programas, aparatos y herramientas tecnológicas con los cuales no todos estaban familiarizados o incluso no tenían acceso, pero que aparecían como el único mecanismo posible para continuar una educación centrada en la enseñanza.

En este sentido, es claro que optar libremente por el homeschooling y verse compelido a realizar las clases remotas como parte de la educación escolar (por una eventualidad como la pandemia) son dos formas diferentes de pensar y responder a los compromisos que supone formar a otros: la primera se centra en el aprendizaje, la segunda en la enseñanza. Este énfasis diferente determina no solo los propósitos de la formación, sino los medios y las acciones a través de las cuales ella se realiza. En el primer caso, el sustento que lo orienta es el de los estilos de aprendizaje que dependen de la singularidad de cada aprendiz, por lo tanto, las necesidades e intereses individuales definen los tiempos y escenarios de aprendizaje. En el segundo caso, las características y condiciones del colectivo etario que reconocemos como infancia, primera infancia o adolescencia, así como la jerarquía y secuencialidad de los contenidos y programas diseñados y aprobados por las instancias gubernamentales que le confieren una suerte de unidad y universalidad al sistema de educación formal en sus diferentes niveles (MARÍN-DÍAZ, 2012).

En todo caso, es claro que la línea que diferencia y separa estos énfasis es cada vez más borrosa, pues en los debates pedagógicos ha adquirido centralidad el concepto de aprendizaje para pensar y actualizar la escuela; esto posibilitó, no solo que el homeschooling sea una alternativa frente a la educación escolar, sino que esta última esté atravesada por el lenguaje del aprendizaje, cuestionando las prácticas de enseñanza en un proceso que se conoce como aprendificación o learnification (BIESTA, 2013; 2016). En general, se puede decir que en el lenguaje de la educación ocurrió un desplazamiento que nos llevó del uso de los conceptos maestro, escuela, 
alumno y enseñanza al uso privilegiado de términos como facilitador, ambiente de aprendizaje, aprendiz y aprendizaje; ello no sólo significó un cambio en el lenguaje, también en las prácticas educativas escolares. En este sentido, se entiende lo que Illich quería destacar cuando afirmó que "la desescolarización de la sociedad implica el reconocimiento de la naturaleza ambivalente del aprendizaje" (ILLICH, 2011, p. 206).

Este pensador austríaco distingue entre el aprendizaje posible en el contexto escolar - unidireccional, rutinario y carente del desarrollo de destrezas - y el que se puede adquirir en otros escenarios de aprendizaje - plurales, diversos y singulares. Al respecto, la diferencia no radicaría en el tipo de aprendizaje posible en cada espacio, todos ellos parecerían iguales en su importancia y características, sino en la naturaleza particular, en las necesidades y los deseos individuales. En otras palabras, lo que se valora es la condición humana de aprendiz permanente, la comprensión del sujeto como una individualidad que, expuesta a diversos ambientes de aprendizaje y competente para aprender, puede disponerse y aprovechar lo que estos ambientes ofrecen según los intereses y necesidades particulares. En el lenguaje del aprendizaje la educación escolar puede ser sustituida por la educación en casa, pues las dos deben ocuparse de conquistar el deseo de aprender del alumno "al mismo tiempo que los conocimientos son pasados de forma amplia, contextualizando con elementos prácticos da vida" (HOMESCHOOLINGBRASIL, s.f.).

La presencia del concepto de aprendizaje no es nueva en el lenguaje de la educación, lo nuevo es su predominio y excesiva centralidad en el campo discursivo de la pedagogía (NOGUERA-RAMÍREZ y MARÍN-DÍAZ, 2019) y la finalidad de ese aprendizaje. En las primeras décadas del siglo $X X$, en lo que hoy conocemos como movimiento de Escuela activa, Escuela nueva o Escolanovismo se discutía la urgencia de atender las necesidades y hacer nacer el deseo en los niños, bajo el entendido que la educación funcional, sería aquella que "toma la necesidad del niño, su interés por alcanzar un fin, como palanca de la actividad que se desea despertar en él" (CLAPARÈDE, 2007, p.41). En este sentido, interés y deseos se formulan como piezas claves para que el infante actúe en determinada dirección (prevista por el adulto) y los adultos son los encargados de proporcionar los elementos y el medio para que dicho aprendizaje ocurra. En la forma actual, el interés y deseo infantil son los que establece la dirección y sentido de la acción educativa y de los aprendizajes, considerando que estos deseos e intereses son innatos y que no requieren más orientación que la voluntad del propio sujeto infantil (MARÍN-DÍAZ, 2013). 
El predominio del concepto aprendizaje y su lenta instalación durante el siglo $\mathrm{XX}$, generó un cuestionamiento sobre las prácticas escolares y puso en tensión los elementos y fines de este cronotopo - un espacio aislado, separado, cerrado orientado por propósitos formativos y centrado en prácticas disciplinares-, con los fines sociales, económicos y culturales que se le asignan en la actualidad -el bienestar y la motivación de los estudiantes, el manejo eficiente de los recursos y la utilidad de sus resultados- (SIMONS y MASSCHELEIN, 2014). Esta situación debilitó su potencia como

[...] un espacio otro; como una esfera espacio-temporal en la cual es posible abandonar (por algunas horas del día o de la semana) los asuntos cotidianos y el entorno conocido para estudiar y practicar saberes, destrezas y experiencias diferentes a aquellos que aparecen en el día a día del mundo. (NOGUERARAMÍREZ y MARÍN-DÍAZ, 2017, p. 615).

El pedido de un retorno gradual con o sin alternancia a la presencialidad indica que las clases remotas no ofrecen lo que se tenía con la educación formal, no solo porque los adultos y los jóvenes no estaban preparados para asumirla y no fue su decisión, sino porque los espacios y las condiciones no estaban dadas para que ella pudiese ocurrir. La espacio-temporalidad que requiere la educación escolar no es posible sin un lugar diferente, sin el distanciamiento físico del mundo familiar y cotidiano que les permita a los jóvenes recibir ese "algo" que la vida corriente no ofrece. Entonces es posible pensar que la escuela, con su tiempo y espacio, brinda unas condiciones particulares para una "experiencia formativa" diferente, esa que permite establecer una relación con el conocimiento heredado por las generaciones anteriores, la práctica de una vida reglar, con normas, disciplina y ejercitación vivida con otros, y la producción de unos modos específicos de ser, estar y construir el mundo (NOGUERA-RAMÍREZ, MARÍN-DÍAZ, 2017).

La experiencia escolar es mucho más que asistir a clases remotas, acompañado o no por los padres, o seguir las directrices definidas por los maestros y la institución escolar; tampoco consiste en mantener rituales como los horarios de clase e incluso usar el uniforme para sentirse "estudiante". Los reclamos de niños y adultos por retornar a la escuela se expresan de diversas maneras. Por ejemplo, algunas movilizaciones recientes claman por la vuelta a la escuela -aunque sus arengas también se confundan con otras exigencias-, también circulan mensajes vehementes a través de redes sociales, como Facebook, acerca del cansancio de los niños frente a sus padres, la sensación de extrañar la escuela, a sus maestros, los recesos escolares y a sus compañeros, o los debates que afrontan algunos padres entre el 
acompañamiento a sus hijos, las demandas laborales y los oficios domésticos-; inclusive, algunos maestros renuncian a su oficio o al menos se sienten tentados a hacerlo, por el agotamiento que genera hablar ante nombres sin cuerpos, privados de la reconvención pedagógica que constituye una mirada en el momento preciso y agobiados por demandas de planeación o seguimiento excesivas. En suma, estos asuntos no constituyen solo síntomas de la contingencia, sino de la particularidad y la necesidad de escuela.

En tanto la escuela está caracterizada por una organización de tiempo y espacio, que se distingue respecto a otros escenarios cotidianos - como el barrio, la calle, los lugares de entretenimiento, entre otros-, permite la constitución de heterotopías, en las que emerge una experiencia diferente. De este modo, la organización que subyace al horario y al calendario escolar, así como a la distribución del aula o del patio de juegos, posibilitan que haya un tiempo de suspensión de las demandas del mundo social (laborales, profesionales, familiares) y que se pueda entrar en contacto con conocimientos que no estarían presentes en un escenario diferente. Esta articulación entre suspensión y profanación -entre otros rasgos que Simons y Masschelein (2014) reconocen en el cronotopo escolar-, serían condición fundamental del carácter público de la institución escolar.

Gracias a que la escuela es un espacio público, es posible que quienes asistan a ella (fundamentalmente los estudiantes) asuman criterios de vida comunes, que si bien son definidos históricamente y pueden variar en el tiempo, también sirven de sustento para construir relaciones sociales que les permiten vivir en sociedad. En este sentido, es importante recordar lo que señalaba Kant (2003) en su curso de pedagogía sobre la importancia de la escuela como institución pública y su relación con la educación en el seno familiar:

\footnotetext{
El fin de tales institutos públicos es el perfeccionamiento de la educación doméstica. Cesarían sus gastos si estuvieran bien educados los padres a los que les ayudan en la educación. En ellos se debe hacer ensayos y educar individuos, y así crearán una buena educación doméstica.

De la educación privada cuidan, o bien los mismos padres, o bien otras personas, que son auxiliares o asalariados, cuando aquéllos no tienen tiempo, habilidad o gusto; pero en la educación dada por éstos, se presenta la dificilísima circunstancia de hallarse la autoridad entre los padres y los ayos. El niño debe regirse por las instrucciones de los ayos y seguir al mismo tiempo los caprichos de los padres. En una educación de esta clase es necesario que los padres cedan toda su autoridad a los preceptores.

¿Pero en qué puede aventajar la educación privada a la pública o ésta a aquella? Parece ser más ventajosa, en general, la educación pública que la privada, no sólo desde el punto de vista de la habilidad, sino también por lo que se refiere al carácter del ciudadano. Es muy frecuente que la educación doméstica no solamente no corrija las faltas de la familia, sino que las aumente (KANT, 2003, p.41-42).
} 
Son claros los riesgos que entraña privatizar o limitar la educación de los niños a las creencias y valores familiares. Ir a la escuela supone el encuentro con otros, es tal vez el primer acto de independencia que viven los infantes y, aunque entraña los peligros de los cuales las familias quieren protegerlos, también entraña las oportunidades que ellos tienen de construir y ensayar las herramientas para estar con otros, para vivir con los diferentes, para pensar y relacionarse con el mundo que les precedió, y con el que se construye con su presencia y en compañía de esos que son sus pares generacionales.

\section{VOLVER A LA ESCUELA ¿UN ENCIERRO POR OTRO?}

[...] la escuela es el lugar y el tiempo para el estudio y para la práctica: actividades escolares que pueden alcanzar un significado y un valor en sí mismas. Pero esto no quiere decir que la escuela, como si fuera una especie de isla o de torre de marfil, se refiera a un tiempo y a un espacio fuera de la sociedad. Lo que se aborda en la escuela está arraigado en la sociedad, en lo cotidiano, pero ha sido transformado por actos sencillos y profundos de suspensión y profanación (temporales). (SIMONS, MASSCHELEIN, 2014, p.41).

Una de las principales críticas que se hace a la escuela es la de ser un lugar de encierro y aislamiento en el que los niños son obligados a tomar distancia de su mundo social y a "perder el tiempo" estudiando temas y contenidos inútiles para resolver los problemas cotidianos y prepararse para el mundo real. Después de casi un año de aislamiento en las casas, solo con el círculo familiar inmediato, no son pocas las voces que reclaman el retorno de los infantes a la escuela, pero ¿por qué volver a la Escuela? ¿por qué salir de un encierro a otro encierro? Dicho de otra forma, ¿qué es lo que no nos gusta del encierro que significa la escuela? y ¿qué es lo que nos ofrece ese encierro?

El encierro que aparece señalado en las críticas a la escuela remite a varios significados. En primer lugar, corresponde a la idea de clausura, represión, reclusión, aislamiento, separación, internación, ordenamiento y disciplinarización no deseables para los cuerpos y las mentes infantiles y juveniles. Aislar sería la manera como se garantiza que cuerpos y mentes informes (sin forma definida) sean formateados, adquieran determinadas conductas y actúen sumisamente, como en el video de The Wall, de Pink Foyd. En segundo lugar, se asume con el propósito de diferenciar, limitar y jerarquizar, encerrar para organizar a los estudiantes en grados escolares y para definir contenidos y asignaturas escolares, independientemente de sus intereses y necesidades, situación evidente en los textos, los contenidos y la forma de evaluación 
con los cuales se trabaja en la escuela. En tercer y último lugar, se refiere a un encierro que distancia del espacio y tiempo real, es anacrónico y no responde a las formas de vida que acompañan la experiencia vital de los infantes y sus medios sociales; en esa medida la escuela propone, reglas, rutinas y actividades que solo son necesarias en ese espacio. Si estos son los sentidos que se le atribuyen al encierro que significa la escuela, tal vez sea necesario preguntarse ¿Cuál es su propósito? ¿Dicho propósito se cumple? ¿Por qué buscar la adquisición de ciertas conductas a través de un mecanismo más bien 'anticuado'?

Lo que probablemente no se identifica en las críticas a la escuela es que se trata de un encierro que permite a los jóvenes participar de un espacio, de unas actividades, de unos conocimientos en las mismas condiciones que lo hacen otros que están con ellos en ese momento, que estuvieron antes o que vendrán después. Es un encierro en el cual las condiciones de inicio se desmarcan de los signos y los rasgos identitarios para que, con su forma de habitar la escuela, cada uno pueda construir con otros un "estar" en el mundo y, a su vez, el mundo en el que puede estar con otros. Es un encierro que habilita aulas, bibliotecas, laboratorios y patios de juegos a los que accede en condiciones de paridad con esos otros, de la misma forma como lo hace con los saberes y conocimientos con los que puede ensayar, con los que se puede equivocar. Es un encierro en que las reglas comunes, antes que formas legales de la norma, son formas de construcción de lo común, de acuerdos y códigos de relacionamiento, explícitos o no, que definen su relación consigo mismo, con los otros y con el medio.

La escuela es una institución anacrónica, que no responde a las formas de vida actuales, marcadas por la flexibilidad, la velocidad, el aprendizaje permanente o la disposición hacia el cambio y la competencia. Tal vez sea esa suerte de anacronismo de la escuela al que los niños y jóvenes quieren volver. Se trata de un encierro que, al aislar, separar y disciplinar, también cuida, prepara, alberga, asiste y protege. Aísla y separa de situaciones sociales que constituyen riesgos vitales para niños y jóvenes, no solo los cuida y protege a ellos, también al mundo que los recibe y el cual deben mantener, transformar y mejorar para su propia existencia. Asimismo, volver y estar en la escuela favorece la disposición para el encuentro con otros mundos. Quizá ello no ocurra a través de la experimentación, pero si mediante el encuentro con libros y otros lenguajes, lo que se traduce en la aproximación a los números, las letras, el lenguaje audiovisual o las formas musicales y reconocer así, las maneras de ser humanos que hemos sido y/o que estamos dejando de ser. Se trata de entender que 
el mundo percibido por nuestros sentidos es ínfimo, si lo contrastamos con la experiencia que nos ofrece el estudio de la producción humana. Además, que el estudio requiere ejercitación, rutinas y ciertos hábitos, que ello demanda distanciarnos de la cotidianeidad de otros espacios sociales, para construir un espacio-tiempo distinto, de suspensión de las demandas del mundo social y de profanación, al entrar en contacto con los conocimientos producidos por otros, en espacios y tiempos diferenciados (SIMONS, MASSCHELEIN, 2014).

En síntesis, cuando se habla de volver a la escuela, de lo que se trata es de retornar a ese cronotopo especial porque allí la vida ocurre de otra forma, se constituye en experiencia, se construyen y tejen nexos con mundos pasados $y$ posibles, se experimenta y ensaya con saberes, útiles y relaciones, pero fundamentalmente se practica y ejercita la vida en contacto con otros. En la escuela no se resuelve la vida ni los problemas sociales concretos de individuos o sociedades, eso le corresponde a otras instituciones; lo que se construye en la institución escolar es una forma de estar con otros en un mundo - también en construcción- y se abre la posibilidad para otros mundos. El retorno a la escuela, la necesidad de volver a ella nos recuerda que la educación es, fundamentalmente, un asunto antropológico, de construcción de lo humano en esa relación que suponen el encuentro con lo extraño, con lo otro, con lo diferente. Por tanto, la exigencia de un regreso a la escuela, después de meses de educación remota, nos ha permitido (re)descubrir la importancia de la tarea educativa de la escuela que, valga decir, todavía es difícil que sea relevada por otras formas de educación o, mejor, de aprendizaje.

\section{REFERENCIAS}

BIESTA, Gert. Devolver la enseñanza a la educación. Una respuesta a la desaparición del maestro. Pedagogía y Saberes, n. 44, p. 119.129, 2016. DOI:

10.17227/01212494.44pys119.129. Disponível em:

https://revistas.pedagogica.edu.co/index.php/PYS/article/view/4069.

BIESTA, Gert. Para além da aprendizagem. Educação democrática para um futuro humano. Belo horizonte: Autêntica, 2013.

CABO, Carlos. El homeschooling en España: descripción y análisis del fenómeno. Tesis para optar al título de Doctor en Pedagogía. España: Universidad de Oviedo, 2012.

CASTELlanOS, Emma y SANCHEZ, Ángela. Proyecto Ley Homeschooling:

Educación en Casa. Colombia: Congreso de la República de Colombia, 2020. 
CLAPARÈDE, Édouard. La educación funcional. España: Biblioteca Nueva. Ministerio de Educación y Ciencia, 2007.

FAIRFIELD, Roy; ROSEN, Summer; JUDSON, Jerome; GREENE, Maxine y PEARL, Arthur. Un mundo sin escuelas. México: Nueva Imagen, 1982.

GUTERSON, David. Family matters: why homeschooling makes sense. New York: Houghton Mifflin Harcourt Publishing Company, 1992.

HOLT, Jhon. How Children fail? Penguin Education, 1995.

HOMESCHOOLINGBRASIL. O que é Homeschooling (Educação Domiciliar)? s.f. Disponível em: https://homeschoolingbrasil.info/o-que-e-homeschooling-educacaodomiciliar/ Consultado: 10 de abril 2021.

ILLICH, Iván. Después de la escuela ¿qué? En: ILLICH, I.; GINTIS, H., GREER, C.; POSTMAN, N.; GROSS, R.; FAIRFIEL, R.; SUMMER, R.; JEROME, J.; GREENE, M. y PEARL, A. Un mundo sin escuelas. México: Nueva Imagen, 1977.

ILLICH, Iván. Obras reunidas I. Fondo de Cultura económica, 2011.

KANT, Immanuel. Pedagogía. España: Akal, 2003.

MARÍN-DÍAZ, Dora. Interés por el gobierno y gobierno a través del interés:

constitución de la naturaleza infantil. Pedagogía y Saberes. Facultad de educación Universidad Pedagógica Nacional. n.37. jul 2013, p. 37-48.

MARÍN DÍAZ, Dora y PARRA LEÓN, Gustavo. El poliedro de los saberes escolares. Praxis \& Saber, Vol. 8 n 17, May 2017, p. 103-123. https://doi.org/10.19053/22160159.v8.n17.2018.7203

MARÍN-DÍAZ, Dora. Interés por el gobierno y gobierno a través del interés:

constitución de la naturaleza infantil. Pedagogía y Saberes, n. 37, p. 37-48, 2012. DOI: $10.17227 / 01212494.37 p y s 37.48$. Disponível em: https://revistas.pedagogica.edu.co/index.php/PYS/article/view/1876.

MARSHALL, Dan y VALLE, James. Public School Reform: Potential Lessons form the Truly Departed. Education Policy Analysis Archives. Vol. 4, n. 12, 1996.

NARODOWSKI, Mariano. y BOTTA, Mayra. La mayor disrupción posible en la historia de la pedagogía moderna: Ivan Illich. Pedagogía y Saberes, Bogotá, n. 46, p. 45.54, junio. 2017.

NOGUERA-RAMÍREZ, Carlos y MARÍN-DÍAZ, Dora. En defensa de la experiencia escolar. Fortalecer las fronteras de la escuela. ETD - Educação Temática Digital, Campinas, SP, v. 19, n. 4, p. 607-621, 2017. DOI: 10.20396/etd.v19i4.8648826. Disponível em:

https://periodicos.sbu.unicamp.br/ojs/index.php/etd/article/view/8648826.

NOGUERA-RAMÍREZ, Carlos y MARÍN-DÍAZ, Dora. La pedagogía considerada como campo discursivo. Pedagogía y saberes. n.50. enero, 2019, p. 29-49. 
ROTHERMEL, Paula. Can We Classify Motives for Home Education? Evaluation and Research in Education. Vol 17, No 2 y 3. School of Education, University of Durham, UK, p. 74-89, 2003.

STEVENS, Mitchell. Kingdom of children: Culture and controversy in the home schooling movement. Princeton, NJ: Princeton University Press, 2001.

SIMONS, Maarten. y MASSCHELEIN, Jan. Defensa de la escuela. una cuestión pública. Buenos Aires: Miño y Dávila, 2014.

VAN GALEN, Jane. Explaining home education: parent's accounts of their decision to teach their own children. The Urban Review, 19 (3), p. 161-177, 1987.

VAN GALEN, Jane. Ideologues and pedagogues: Parents who teach their children at home. In: VAN GALEN, Jane y PITMAN, Mary. (Eds.). Home schooling: Political, historical, and pedagogical perspectives. Norwood, NJ: Ablex, p. 63-76, 1991.

\section{NOTAS}

DE REGRESO A CASA: HOMESCHOOLING VERSUS EDUCACIÓN ESCOLAR

Back home: Home education versus school education

De volta para casa: ensino doméstico versus educação escolar

\section{Dora Lilia Marín-Díaz \\ Doctora en Educación Facultad de Artes Universidad Distrital Francisco José de Caldas Bogotá, Colombia \\ dlmarind@udistrital.edu.co (1) https://orcid.org/0000-0002-0466-3368 \\ Ana Cristina León-Palencia Magister en Educación \\ Facultad de educación \\ Universidad Pedagógica Nacional Bogotá, Colombia acleon@pedagogica.edu.co \\ (D) https://orcid.org/0000-0001-7489-0681}

Endereço de correspondência do principal autor

Carrera 58 No. 80-81 interior 2apartamento 413, Cundinamarca. Bogotá DC, Colombia. Carrera 27 No. 61C-20 Apto. 402, Cundinamarca. Bogotá DC, Colombia.

\section{AGRADECIMENTOS}

Não se aplica.

CONTRIBUIÇÃO DE AUTORIA

Concepção e elaboração do manuscrito: D. L. Marín-Díaz, A. C. León-Palencia

Coleta de dados: D. L. Marín-Díaz, A. C. León-Palencia

Análise de dados: D. L. Marín-Díaz, A. C. León-Palencia

Discussão dos resultados: D. L. Marín-Díaz, A. C. León-Palencia

Revisão e aprovação: D. L. Marín-Díaz, A. C. León-Palencia

\section{CONJUNTO DE DADOS DE PESQUISA}

O conjunto de dados que dá suporte aos resultados deste estudo não está disponível publicamente. 


\section{FINANCIAMENTO}

Não se aplica.

\section{CONSENTIMENTO DE USO DE IMAGEM}

Não se aplica.

\section{APROVAÇÃO DE COMITÊ DE ÉTICA EM PESQUISA}

Não se aplica.

\section{CONFLITO DE INTERESSES}

Não se aplica.

LICENÇA DE USO - uso exclusivo da revista

Os autores cedem à Zero-a-Seis os direitos exclusivos de primeira publicação, com o trabalho simultaneamente licenciado sob a Licença Creative Commons Attribution (CC BY) 4.0 International. Esta licença permite que terceiros remixem, adaptem e criem a partir do trabalho publicado, atribuindo o devido crédito de autoria e publicação inicial neste periódico. Os autores têm autorização para assumir contratos adicionais separadamente, para distribuição não exclusiva da versão do trabalho publicada neste periódico (ex.: publicar em repositório institucional, em site pessoal, publicar uma tradução, ou como capítulo de livro), com reconhecimento de autoria e publicação inicial neste periódico.

PUBLISHER - uso exclusivo da revista

Universidade Federal de Santa Catarina. Núcleo de Estudos e Pesquisas da Educação na Pequena Infância - NUPEIN/CED/UFSC. Publicação no Portal de Periódicos UFSC. As ideias expressadas neste artigo são de responsabilidade de seus autores, não representando, necessariamente, a opinião dos editores ou da universidade.

EDITORES - uso exclusivo da revista Márcia Buss-Simão e Kátia Agostinho.

HISTÓRICO - uso exclusivo da revista

Recebido em: 04-05-2021 - Aprovado em: 18-06-2021 